\title{
Ansiedad al tratamiento estomatológico y factores socio-demográficos: Estudio con adultos.
}

\author{
Dental anxiety and factors \\ socio-demographics: study with adults.
}

Aceptado para su publicación

09 de mayo de 2013

Ansiedade ao tratamento odontológico e

fatores sociodemográficos: estudo com adultos.

\section{Resumen}

Maestría en Educación y Ciencias. Profesora e investigadora del Grupo Atención a la Salud Individual y Colectiva, Curso de Odontología de la Universidad del Vale do Itajaí, Santa Catarina, Brasil.E-mail: erabaldo@univali.br

\section{Constanza Marin} Doctora en Periodoncia. Profesora e investigadora del Grupo Atención a la Salud Individual y Colectiva, Curso de Odontología de la Universidad del Vale do Itajaí, Santa Catarina, Brasil.

E-mail: constanza@geoforma.com.br

\section{Silvana Marchiori de Araújo}

Doctora en Odontopediatría. Profesora e investigadora del Grupo Atención a la Salud Individual y Colectiva, Curso de Odontología de la Universidad del Vale do Itajaí, Santa Catarina, Brasil. E-mail: silmarchiori@univali.br

Dirección para correspondencia Elisabete Rabaldo Bottan Av. Atlântica 1020, ap. 1801 Balneário Camboriu - Santa Catarina - Brasil CEP: 88330-006 - Fono/Fax: (55) 47334 I 7564 -E-mail: erabaldo@univali.br
El objetivo fue verificar la correlación entre ansiedad al tratamiento estomatológico y factores socio-demográficos (género y edad). El estudio se caracteriza como descriptivo, con muestra no probabilística formada por sujetos con edad igual o superior a 30 años, en situación no clínica. Fue aplicado un cuestionario con datos sobre edad, género, frecuencia y motivos de la consulta estomatológica. Para determinarse la ansiedad, fue utilizada una adaptación de la escala de Corah (Dental Anxiety Scale - DAS). Participaron de la investigación 287 sujetos (54\% hombres; $46 \%$ mujeres). Las edades variaron de 30 a 84 años. La puntuación media del grado de ansiedad fue de 7,4 puntos. Hombres y sujetos más jóvenes tuvieron frecuencia más elevada para ansiedad. En los dos últimos años, $70,8 \%$ realizaron por lo menos una consulta estomatológica teniendo como principal motivo la consulta de rutina. La consulta en intervalo de tiempo mayor (6 o más años) fue más frecuente entre los individuos con alto $(\mathrm{I} I, \mathrm{I} \%)$ y exacerbado $(87,5 \%)$ nivel de ansiedad. Con base en el cálculo del chi-cuadrado, se concluye que hubo una relación significativa entre edad y grado de ansiedad, sin embargo, entre género y grado de ansiedad no hubo relación significativa.

Palabras clave

Ansiedad al Tratamiento Estomatológico; Relaciones Dentista-Paciente; Salud Oral; Adulto. 
REVISTA FACULTAD DE ODONTOLOGÍA ISSN No 1668-7280 - Vol. VI No 1 - 2013
INVESTIGACIÓN

Rabaldo Bottan - Marin - Marchiori de Araújo

\section{Abstract}

The objective was to verify the association with socio-demographic factors (gender and age) and dental anxiety. The study was characterized as descriptive, with no random sample consisting of subjects with age less than 30 years in clinical situation. A questionnaire with items about age, gender, frequency and reasons for the dental appointment was applied. To determine the anxiety was used a modified Corah Scale (Dental Anxiety Scale - DAS). A total of 287 subjects participated of the inquiry ( $54 \%$ male, $46 \%$ women). The ages ranged from $30-84$ years. The average result of the degree of anxiety was 7.4 points. Men and younger subjects had higher prevalence for anxiety disorders. In the past two years, $70.8 \%$ had at least one dental appointment with the main reason the routine visit. The dental appointment in greater time interval $(6$ or more years) was more frequent between the individuals with stop (II.1\%) and exacerbated (87.5\%) level of anxiety. Based on the calculation of chisquare, it is concluded that there was a significant relationship between age range and level of anxiety, however, between gender and anxiety levels there weren't significant relationship.

\section{Keywords}

Dental Anxiety; Dentist-Patient Relations; Oral Health; Adult.

\section{Resumo}

O objetivo da pesquisa foi verificar a relação entre ansiedade ao tratamento odontológico e fatores sociodemográficos (gênero e idade). O estudo caracteriza-se como descritivo, com amostra não probabilística constituída por sujeitos com idade igual ou superior a 30 anos, em situação não clínica. Foi aplicado um questionário com itens sobre idade, gênero, freqüência e motivos da consulta odontológica. Para se determinar a ansiedade, foi utilizada uma adaptação da Dental Anxiety Scale Corah (DAS). Participaram da investigação 287 sujeitos ( $54 \%$ homens; $46 \%$ mulheres), com idades entre 30 e 84 anos. 0 escore médio do grau de ansiedade foi de 7,4 pontos. Homens e sujeitos mais jovens tiveram frequência mais elevada para ansiedade. Nos dois últimos anos, 70,8\% efetivaram pelo menos uma consulta odontológica tendo como principal motivo a consulta de rotina. A consulta em intervalo de tempo maior foi mais frequente entre os indivíduos com elevado nível de ansiedade. Concluiu-se, com base no cálculo do qui-quadrado, que houve uma relação significativa entre faixa etária e grau de ansiedade, porém entre gênero e grau de ansiedade não houve relação significativa.

\section{Palavras chave}

Ansiedade ao Tratamento Odontológico; Relações Dentista-Paciente; Saúde Bucal; Adulto.

\section{Introducción}

Cuidar de la salud oral es primordial a todo individuo, independiente de edad, nivel de escolaridad o clase social. Todavía, gran parte de la población, principalmente de nivel socioeconómico más bajo, enfrenta dificultades para acceder a los servicios odontológicos, en virtud del alto costo de los servicios particulares y de la indisponibilidad de determinados procedimientos en las unidades básicas de salud pública y también por la carencia de informaciones sobre educación para la salud. Estos obstáculos, además de otros, indiscutiblemente, interfieren en la calidad de la salud oral de las personas.

Un factor que ejerce gran influencia en el comportamiento en cuanto a los cuidados de la salud oral y que, a veces, no es considerado, es la ansiedad al tratamiento estomatológico. La ansiedad puede ser considerada como un significativo indicador de una precaria condición de salud oral y de bajos índices de calidad de vida; probablemente, por esto sea una constante preocupación de las investigaciones, en el área estomatológica. Según Smith y Heaton', la ansiedad al tratamiento estomatológico es un fenómeno que ha sido bien estudiado desde el final de los años sesenta, en el siglo pasado, y que merece continuar siendo analizado.

El impacto emocional, los miedos y las fantasías generadas por el tratamiento estomatológico deben ser considerados por el profesional, pues la forma por la cual la persona elabora internamente esa experiencia es decisiva en la formación de sus expectativas y reacciones frente a la con- 
sulta estomatológical-3. Por lo tanto, el manejo de pacientes temerosos es considerado un gran desafío para los estomatólogos.

Actualmente, los procedimientos odontológicos, con equipamientos sofisticados y perfeccionamiento de las técnicas permiten la reducción significativa de la incomodidad del paciente, sin embargo la ansiedad persiste. Entre 6 y $15 \%$ de la población adulta manifiesta algún grado de ansiedad al tratamiento estomatológico lo que puede inducir a la cancelación o postergación de la consulta, acarreando efectos negativos para la salud $d^{4-5}$.

De allí la importancia de desarrollar investigaciones sobre esta temática para proveer elementos que orienten a los profesionales del área odontológica en cuanto a las reacciones del paciente al tratamiento. Esta investigación tuvo como propósito describir la frecuencia de ansiedad al tratamiento estomatológico y verificar su asociación con género y edad en sujetos adultos.

\section{Materiales y métodos}

Se trata de un estudio descriptivo, transversal, mediante recolección de datos primarios. La población objetivo de estudio constó de sujetos adultos, que estaban participando de la Semana de Salud Oral, de la municipalidad de Itajaí (Santa Catarina/Brasil), en octubre de 2010.

La Semana de Salud Oral es desarrollada, anualmente, cuando son realizadas innúmeras actividades educativas y preventivas para la población. Estas acciones resultan de un trabajo conjunto entre la carrera de Odontología de la Universidad del Vale do Itajaí - UNIVALI, la Alcaldía Municipal de Itajaí y la Asociación Brasileña de Odontología. Una de las actividades de esta Semana sucede en la plaza principal de la municipalidad, ubicada en el centro de la ciudad, y éste fue el lugar donde se buscó a la población de esta investigación.

Fue obtenida una muestra no probabilística, por conveniencia, teniendo como criterios de inclusión: a) tener condiciones físicas y mentales para comprender y responder las preguntas del instrumento de recolección de datos; b) poseer edad igual o superior a 30 años; c) aceptar, por libre arbitrio, participar de la investigación, firmando el Término de Consentimiento Libre y Aclarado. El instrumento para recolección de datos fue un cuestionario con siete preguntas, así distribuidas: dos para caracterización de los sujetos (género y edad); cuatro para determinación del nivel de ansiedad; $y$ una para identificación de la frecuencia y motivos de la consulta estomatológica.

Para la clasificación del nivel de ansiedad, las preguntas y los criterios fueron adaptados de la escala Dental Anxiety Scale (DAS) ${ }^{6}$. En la versión original, la DAS está compuesta por cuatro preguntas, con cinco alternativas de respuestas en cada una. Para esta investigación, se redujeron a cuatro las alternativas de respuestas. Con esta adaptación, la clasificación de los sujetos según la ansiedad al tratamiento estomatológico quedó así distribuida: hasta 04 puntos - sin ansiedad; de 05 a 08 puntos - bajo nivel de ansiedad; de 09 a 12 puntos - alto nivel de ansiedad; de 13 a 16 puntos - exacerbado nivel de ansiedad.

La aplicación del instrumento fue realizada por cinco alumnos de Odontología que fueran trenados previamente. Los colectores efectuaban la lectura y explicación de cada una de las preguntas, individualmente a aquellos sujetos que aceptaron participar de la investigación. Las respuestas expresadas por los sujetos eran registradas en el instrumento.

Los datos fueron tabulados obteniéndose la distribución de la frecuencia (relativa y absoluta) para cada una de las variables en análisis (género, edad, nivel de ansiedad, frecuencia y motivos de la consulta estomatológica). Posteriormente, por el test estadístico no paramétrico del chi-cuadrado (nivel de significancia de 5\%), se determinó la significación de la influencia de género y edad en el grado de ansiedad al tratamiento estomatológico. Para el cálculo de chi-cuadrado, se agruparon los sujetos del nivel alto y exacerbado.

El proyecto de la pesquisa fue aprobado por la Comisión de Ética en Investigación de la UNIVALI, con protocolo $\mathrm{n}^{\circ}$ 00 I4.0.223.000.

\section{Resultados}

El grupo investigado estuvo constituido por 287 sujetos; $53,9 \%$ eran del género masculino y $46,1 \%$ del femenino. Las edades variaron de 30 a 84 años; 50,6\% estaban incluidos en la edad de 5 I o más años y $49,4 \%$ entre 30 y 50 años.

En cuanto al nivel de ansiedad, 74, I\% fueron clasificados como portadores de ansiedad. La puntua- 
ción media entre los sujetos del género femenino fue 7,I puntos; entre los hombres, fue 7,7 puntos. La media del grupo fue de 7,4 puntos, lo que clasifica la muestra como portadora de bajo nivel de ansiedad. La distribución de la frecuencia para cada nivel de ansiedad se encuentra en la Figura I.

aSin ansiedad $\square$ Bajo nivel $\square$ Alto nivel Exacerbado nivel

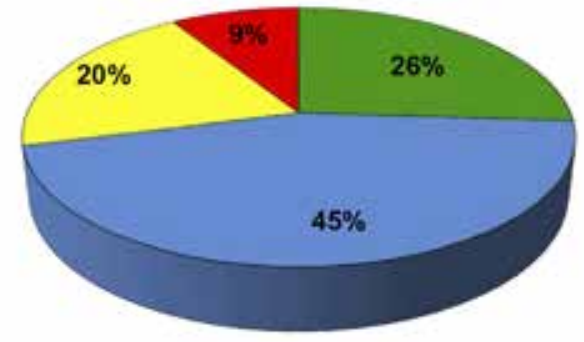

Figura I. Frecuencia relativa de las clases que componen los grados de ansiedad en el grupo investigado.

Entre los portadores de ansiedad, el porcentaje de hombres $(56,1 \%)$ fue superior al de mujeres $(43,9 \%)$. El comportamiento entre los géneros, cuando se hace el análisis según los diferentes niveles de ansiedad, fue similar en todos ellos, excepto para el nivel exacerbado en que la diferencia fue acentuada, con una relación de 3 hombres para I mujer (Figura 2). Todavía por el test del chi-cuadrado $(p=0,5996)$, el género no ejerció influencia significativa en la determinación del grado de ansiedad.

La relación entre nivel de ansiedad y edad evidenció que en el grupo con $5 \mathrm{I}$ o más años la frecuencia de portadores de ansiedad es menor, cuando comparado con el grupo de 30 a 50 años de edad

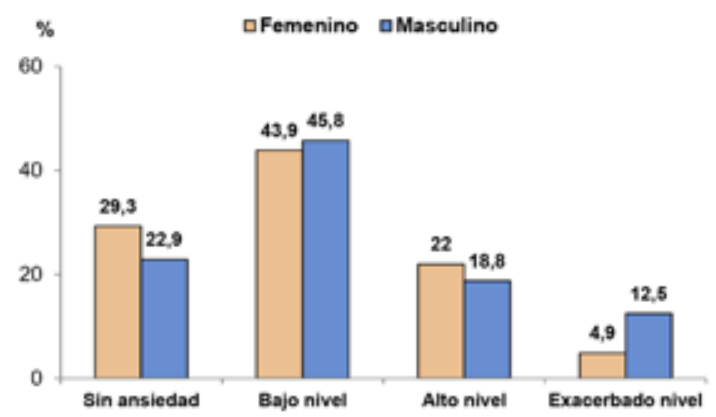

Figura 2. Frecuencia relativa de las clases que representan los diferentes niveles de ansiedad, en el grupo investigado, según el género.

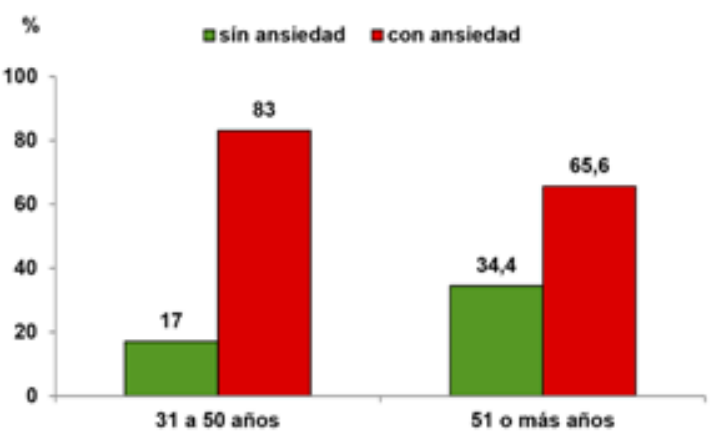

Figura 3. Frecuencia relativa de las clases que indican presencia o no de ansiedad, en el grupo investigado, según la edad.

(Figura 3). Esta diferencia, en conformidad con el test chi-cuadrado $(p=0,0094)$, es significativa, o sea, la edad ejerció influencia significativa en la determinación del grado de ansiedad.

La mayoría $(70,8 \%)$ realizó por lo menos una consulta estomatológica en el período de dos o menos años, en relación a la fecha de la colecta de datos (Figura 4).

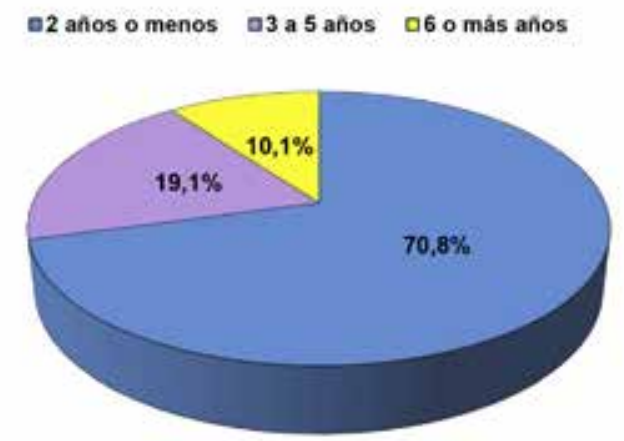

Figura 4. Frecuencia relativa de las clases que componen el intervalo de tiempo en relación a la última consulta efectuada.

La frecuencia de la consulta en función del nivel de ansiedad indica que los intervalos de tiempo mayores están entre los sujetos de alto y exacerbado niveles de ansiedad (Figura 5).

Los principales motivos para realización de la consulta, según los investigados, fueron: consulta de rutina, dolor, problemas gingivales y caries.

\section{Discusión}

La relación entre ansiedad y fuga de la consulta estomatológica es enfáticamente explicada por 


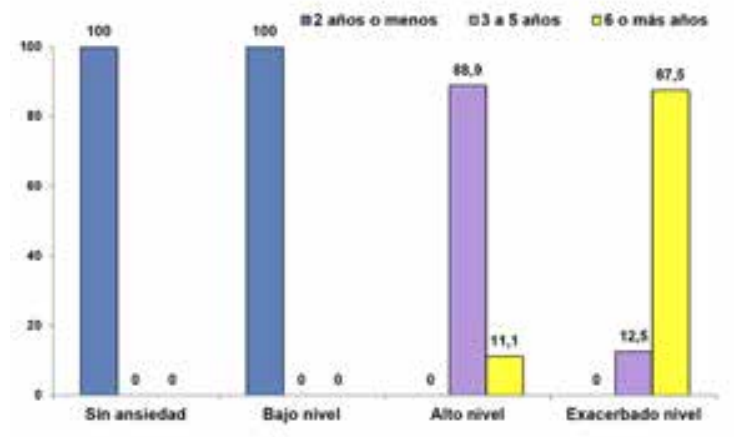

Figura 5. Frecuencia relativa de las clases que componen el intervalo de tiempo en relación a la última consulta efectuada, según el nivel de ansiedad.

diversos autores I-I0. En el grupo que investigamos, los sujetos con alto y exacerbado nivel de ansiedad constituían la mayoría de aquellos que realizaban consulta en intervalos de tiempo superiores a tres años $y$, generalmente, en situaciones de dolor, esto se relaciona con lo encontrado en la literatura cuando explica que la ansiedad actúa como una de las barreras a la búsqueda de atención estomatológical-1!.

Por otro lado, $70,8 \%$ de los integrantes de esta investigación afirmaron haber realizado, rutinariamente, consulta en los últimos dos años. Probablemente, este hecho haya contribuido para que la mayoría de los inquiridos se clasifique como portador de bajo nivel o sin ansiedad. Parece que frecuentar la consulta estomatológica favorece la reducción de la ansiedad y estimula la búsqueda y mantenimiento de estándares adecuados de salud oral.

Los síntomas de la ansiedad son semejantes, en diferentes regiones del mundo, pero el impacto causado por ella varía de acuerdo con el significado sociocultural, por lo que se torna difícil establecer una rígida comparación entre las diferentes investigaciones. Además de que, en estas investigaciones hay diferencias en cuanto a los instrumentos de clasificación de los niveles de ansiedad, a los procedimientos de definición de la muestra y a la condición de los sujetos (en tratamiento o no), entre otras.

A pesar de esas limitaciones, se puede afirmar que la ansiedad en relación al tratamiento estomatológico es una realidad con la cual, aún, muchas personas conviven. La prevalencia de este tipo de comportamiento entre adultos es de más de
$20 \%$, en países con sistema de atención bucal bien estructurado $^{12}$. En esta investigación, el porcentaje de sujetos que manifestaron algún grado de ansiedad fue de $74,1 \%$.

Algunos factores socio demográficos, entre los que se destacan el género, edad y escolaridad, son apuntados como influyentes en los niveles de ansiedad al tratamiento estomatológico. En la mayoría de los estudios, se observó que las mujeres son más ansiosas que los hombres s, $^{50-15}$. Esta diferencia puede ser debida a la formación cultural de diversas sociedades, donde la mujer, comparada a los hombres, consigue expresar, sin mucho prejuicio, sus temores $y$, también, porque el número de mujeres que busca atención estomatológica es mayor que el de hombres ${ }^{14,16}$.

En nuestra investigación, aunque estadísticamente el género no haya ejercido una influencia significativa, se encontró una condición distinta de aquella apuntada en un gran número de investigaciones. El mayor porcentaje de sujetos ansiosos fue de hombres, probablemente, este resultado esté relacionado con la composición de la muestra, que fue obtenida por conveniencia resultando un número de sujetos del género masculino un poco más elevado que el número de mujeres.

Concerniente a la influencia de la edad, la literatura presenta posicionamientos diferentes. Hay estudios refiriendo que, con el aumento de la edad, las personas se tornan más temerosas por haber convivido con un mayor número de tratamientos ${ }^{14,17,18}$. Otros ${ }^{4,5,8}$ afirman que la ansiedad declina con el pasar de los años, situación ésta, también identificada en esta pesquisa.

A pesar del gran volumen de material publicado, el reconocimiento y la caracterización precisa de pacientes ansiosos no es una tarea fácil. Por eso, es fundamental la atención del estomatólogo, para con los aspectos psicosociales referentes a su paciente, pues las experiencias derivadas de la relación profesional-paciente son importantes para la determinación de la ansiedad al tratamiento estomatológico.

Teniendo en cuenta el impacto negativo que la ansiedad puede ejercer sobre el cuidado dental y la calidad de vida de las personas, es necesario que el estomatólogo entienda que hasta hoy este comportamiento persiste entre algunas personas. Así, los profesionales deben investigar las preocupaciones de sus pacientes, antes de iniciar 
el tratamiento.

La gestión eficiente de la ansiedad al tratamiento dental es de suma importancia y debe consistir en un enfoque múltiple. Hay una gama de opciones que pueden ser empleadas para controlar la ansiedad, que son: los enfoques no farmacológicos (comunicación, gestión de comportamiento) y los farmacológicos (sedación, anestesia general y sedación de la inhalación). En la prevención debe tenerse en cuenta que es fundamental la conversa con el paciente, que se debe informar lo que será realizado y que se mantenga una relación dentista-paciente cordial| $\left.\right|^{14,19-20}$.

\section{Conclusiones}

A partir de los resultados obtenidos se concluye que el grupo investigado se clasifica como portador de nivel bajo de ansiedad al tratamiento estomatológico y existió una relación estadísticamente significativa entre la edad y nivel de ansiedad. Hubo ausencia de relación estadísticamente significativa entre sexo y ansiedad.

\section{Referencias}

I. Smith TA, Heaton LJ. Fear of dental care: are we making any progress? J Am Dent Assoc 2003; 134 (8): I I0I-8.

2. Márquez-Rodríguez JA, Navarro-Lizaranzu MC, Cruz-Rodríguez D, Gil-Flores J. Por qué se tiene miedo al dentista? Rev Actual Odontoestomatol Esp 2004; 9 (2): 165-74.

3. Armfield JM. Towards a better understanding of dental anxiety and fear: cognitions vs. experiences. Eur. j. oral sci. 2010; I 8 (3): 259-64.

4. Åstrøm AN, Skaret E, Haugejorden O. Dental anxiety and dental attendance among 25-yearolds in Norway: time trends from 1997 to 2007. BMC oral health 20 I I; II (I): I0.

5. Enkling N, Marwinski G, Jörhen P. Dental anxiety in a representative sample of residents of a large German city. Clin. oral investig. 2006; 10 (I): 84-91.

6. Corah NL. Development of a dental anxiety scale. J. dent.res. 1969; 48: 59.

7. $\mathrm{Ng}$ SK, Leung WK. A community study on the relationship of dental anxiety with oral health status and oral health-related quality of life. Community Dent Oral Epidemiol 2008; 36 (4): 347-56.

8. Crofts-Barnes NP; Brough E; Wilson KE; Beddis
Aj; Girdler NM. Anxiety and quality of life in phobic dental patients. J Dent Res 2010; 89 (3): 302-6.

9. Crocombe LA, Broadbent JM, Thomson WM, Brebnnan DS, Slade GD, Poulton R. Dental visiting trajectory patterns and their antecedents. J. public health dent. 20I I; 7I (I): 23-3I.

10. Eitner S, Wichmann M, Paulsen A, Holst S. Dental anxiety: an epidemiological study on its clinical correlation and effects on oral health. J Oral Rehabil 2006; 33 (8): 588-93.

II. Farah G]. Como controlar a ansiedade do paciente frente a procedimentos odontológicos. Rev. Dental Press Periodontia Implantol. 2010; 4 (2): 33-4.

12. Maniglia-Ferreira C, Gurgel-Filho ED, BöneckerValverde G, Moura EH, Deus G, Coutinho-Filho T. Ansiedade odontológica: nível, prevalência e comportamento. RBPS 2004; I7 (2): 5I-5.

13. Heft MW, Meng X, Bradley MM, Lang PJ. Gender differences in reported dental fear and fear of dental pain. Community dent. oral epidemiol. 2007; 35 (6): $421-8$.

14. Lima-Alvarez M, Casanova-Rivero, Y. Miedo, ansiedad y fobia al tratamiento estomatológico. Rev Hum Med 2006; 6 (I): 0-0.

15. Malvania EA, Ajithkrishnan CG. Prevalence and socio-demographic correlates of dental anxiety among a group of adult patients attending a dental institution in Vadodara city, Gujarat, India. Indian j. dent. Res. 20I I; 22 (I): I79-80.

16. Machin R, Couto MT, Silva GSN, Schraiber LB, Gomes R, Figueiredo WS, Valença AO, Pinheiro TF. Concepções de gênero, masculinidade e cuidados em saúde: estudo com profissionais de saúde da atenção primária. Ciênc. saúde coletiva 20II; I6 (I I): 4503-I2.

17. Kumar S, Bhargav $P$, Patel A, Bhati $M$, Balasubramanyam G, Duraiswamy $P$ et al. Does dental anxiety influence oral health-related quality of life? Observations from a cross-sectional study among adults in Udaipur district, India. J Oral Sci 2009; 5 I (2): 245-54.

18. Carvalho RWF, Vasconcelos BCE, Cardoso MSO, Pereira MAS, Pereira JC, Bastos AS et al. Ansiedade frente ao tratamento odontológico: prevalência e fatores preditores em brasileiros. Cienc Saude Colet 2012; 17 (7): 1915-22.

19. Sharif MO. Dental anxiety: detection and management. J. Appl. Oral Sci. [on line] 2010; 18 (2): i-i.

20. Silva AM, Santos CC, Regiori MG, Andia-Merlin $R$, Alegretti CE, Okida Y. et al. Study of emotional and psychological factors that may interfere in the dental treatment. J. Health Sci. Inst. 2009; 27 (3): 249-53. 